\title{
Imaturos de Culicidae (Diptera) encontrados em recipientes instalados em mata residual no munícipio de Londrina, Paraná, Brasil
}

\author{
João A. C. Zequi ${ }^{1}$ José Lopes ${ }^{2}$ \& Ísis M. Medri ${ }^{2,3}$ \\ 1 Programa de Pós-Graduação em Agronomia, Departamento de Biologia Animal e Vegetal, Centro de Ciências Biológicas, \\ Universidade Estadual de Londrina. E-mail: zequi@uel.br \\ 2 Departamento de Biologia Animal e Vegetal, Universidade Estadual de Londrina. Rodovia Celso Garcia Cid, PR 445 Km 380, \\ Campus Universitário, Caixa Postal 6001, 86051-990 Londrina, Paraná, Brasil. E-mail: jea@uel.br \\ 3 Programa PIBIC/CNPQ, Universidade Estadual de Londrina.
}

\begin{abstract}
Immature specimens of Culicidae (Diptera) found in installed recipients in forest fragments in the Londrina, Paraná, Brazil. Forest fragments in an urban area were found to be a situable site for Culicidae breeding. This research aims to inventory the Culicidae species that use artificial breeding sites, their coexistence, and the population variation in a fragment forest in the Londrina City, Paraná State. Biweekly collecting efforts were performed from October 1995 to September 1996 using tires and bamboo internodes traps installed at a ground level and bamboo internodes traps at two $2 \mathrm{~m}$ height. A total of 12,656 culicid specimens belonging to five genus and 11 species were collected. The most abundant species in those artificial breeding sites were Limatus durhami Theobald, 1901, Culex eduardoi Couple \& Garcia, 1968, Aedes terrens (Walker, 1856), Culex quinquefasciatus Say, 1823 and Aedes albopictus. (Skuse, 1894). The greatest number of individuals was collect in tires and Limatus durhami and Aedes terrens, showed a preference to the breeding sites. The capture of Aedes aegypti (Linnaeus, 1762), Aedes albopictus and Haemagogus leucocelaenus (Dyar \& Shannon, 1924) in different collections efforts indicates that this kind of environment must be taken in account in the vector control actions and constantly monitored.
\end{abstract}

KEY WORDS. Bamboo, breeding sites, forest fragments, tire, vectors.

RESUMO. Fragmentos de mata na área urbana ou periurbana podem ser locais favoráveis a procriação de Culicidae. Esse trabalho teve como objetivo levantar as espécies de culicídeos que utilizam criadouros artificiais, suas coexistência e as flutuações populacionais em uma reserva de mata localizada em Londrina - PR. Realizouse coletas quinzenais de outubro de 1995 a setembro de 1996 em pneus e internódios de bambu armadilha que foram instalados ao nível do solo e outros internódios instalados a $2 \mathrm{~m}$ de altura. Coletou-se 12.656 espécimes, pertencentes a cinco gêneros e 11 espécies. As espécies mais abundantes nos criadouros foram Limatus durham Theobald, 1901, Culex eduardoi Casal \& Garcia, 1968, Aedes terrens (Walker, 1856), Culex quinquefasciatus Say, 1823 e Aedes albopictus (Skuse, 1894). O maior número de indivíduos foi coletado em pneus, sendo que Limatus durham e Aedes terrens, apresentaram preferência nesse criadouro. A presença de Aedes aegypti (Linnaeus, 1762), Aedes albopictus e Haemagogus leucocelaenus (Dyar \& Shannon, 1924) em diferentes coletas indica que esse tipo de ambiente não deve ser ignorado nas ações de controle de vetores, merecendo constante monitoramento.

PALAVRAS CHAVE. Bambu, criadouro, fragmentos de mata, pneu, vetores.

Poucas pesquisas sobre a diversidade de Culicidae têm sido realizadas no interior de parques ou outro tipo de áreas de preservação, dentro do perímetro urbano ou periurbano, que contenham fragmentos de mata. Dentre os trabalhos relacionados, consta o de Lopes et al. (1993), BARBosa et al. (1993), ForatTinI et al. (1993), Guimarães et al. (2000), UrbinatTi (2001), Taipe-Lagos \& Natal (2003) e Paterno \& Marcondes (2004). A cidade de Curitiba, no Estado do Paraná, caracteriza-se por ser uma das cidades brasileiras com maior área de fragmentos de mata na região urbana e, paralelamente, vários trabalhos com Culicidae têm sido também realizados (SILVA \& Lozovei 1996, 1998, 1999, Silva et al. 2004, Calado \& Silva 2001, Barbosa et al. 2003).

Estes ambientes, se não monitorados, tornam-se locais de descartes de lixo, pneus usados e recipientes diversos, alguns podendo tornar-se criadouros artificiais para espécies oportunistas de Culicidae. Estas condições de mata, com recipiente que retém água, podem significar refúgios de espécies de mosquitos se, durante as operações de combate a estes insetos, não

Revista Brasileira de Zoologia 22 (3): 656-661, setembro 2005 
forem incluídas nas estratégias operacionais. Além da sua importância sanitária, é significativa a importância ecológicaevolutiva, do aproveitamento de recipientes com água, pelas fêmeas de Culicidae, para a procriação.

Além dos objetos descartados para o interior destas pequenas reservas de matas, também são detectados criadouros naturais de Culicidae. De acordo com o hábito das espécies, estas podem depositar seus ovos, em ocos de árvores (Jenkins \& Carpenter 1946), Sota et al. 1994); nas axilas de plantas, em conchas de moluscos (ForatTini 2002), em bromélias (ForatTini \& Marques 2000), em entrenós de bambu e taquara (Sota \& MogI 1996, Lozover 1998, Zequi \& LoPes 2001), nas nascentes de água ou nos ribeirões existentes em seu interior (Consolim et al. 1993, Lopes \& Lozovei 1995, Quintero et al. 1996, Lopes et al. 2002).

A presente pesquisa visou verificar a existência de espécies silvestres que tenham potencial para sobreviverem em capões isolados de mata em área urbana, se reproduzirem em criaduros artificiais e diagnosticar as suas coexistências.

\section{MATERIAL E MÉTODOS}

A pesquisa foi realizada em uma reserva florestal conhecida como Mata Daher. Ocupa uma área de 3,3 hectares, fica localizada na área urbana de Londrina, Paraná, Brasil, próximo a rodovia Celso Garcia Cid, PR 445, Km 380 (2318'55"S; $\left.51^{\circ} 12^{\prime \prime} 16^{\prime \prime} \mathrm{W}\right)$. É uma mata alterada, onde ainda existem árvores nativas de grande porte. No estrato inferior, como vegetação secundária, encontra-se grande quantidade de cipós e abundância de taquara do gênero Merostachys Spreng, 1824. A área, caracteriza-se por uma topografia elevada, com altitude de 573 metros estando em platô, margeada ao norte pelo ribeirão Cambézinho e ao sul por uma pequena nascente de água.

Próximo à região central da mata foram instalados três pneus de automóvel, ao nível do solo, na posição vertical e amarrados ao tronco de árvores. Os pneus ficaram em linha reta e distantes $10 \mathrm{~m}$ um do outro. Paralelamente a linha de disposição dos pneus e, a 10 metros de distância lateral, foram instaladas ao nível do solo três internódios-armadilhas de bambu, da espécie Bambusa sp. Schreb (Bambuseae, Poaceae). Em outra linha paralela, outras três internódios-armadilhas de Bambusa sp. foram fixados em troncos de árvores, a $2 \mathrm{~m}$ de altura.

Os internódios-armadilhas caracterizam-se como larvitrampa, onde um internódio de bambu é separado do colmo, mantendo-se a região inferior fechada pelo nó e a parte superior aberta. Este tipo de criadouro artificial é amplamente conhecido na literatura especializada. Introduz-se aqui, o termo internódio-armadilha para facilitar a discussão e diferenciar de outras situações estudadas da colonização dos internódios naturais, constituintes do colmo integro da planta e em seu local original de florescência.

Os internódios-armadilhas mediam aproximadamente $30 \mathrm{~cm}$ de altura, por $7,5 \mathrm{~cm}$ de diâmetro e com capacidade aproximadamente de 1,4 l. Em todos os recipientes foi introduzida água destilada. As coletas foram realizadas a cada quinze dias, de outubro de 1995 a setembro de 1996.

Durante as coletas quinzenais, a água do criadouro foi coada, retirando-se todas as larvas e em seguida devolvida para o recipiente. Quando necessário, o volume inicial foi completado com água destilada.

No laboratório, as larvas foram criadas até obtenção do quarto instar. As pupas e uma amostra de larvas foram criadas até a obtenção do inseto adulto. As identificações foram realizadas a partir de larvas e exúvias de quarto instar e confirmada pelos respectivos adultos. Para esta identificação utilizou-se as chaves de LANe (1953) e Forattini (2002).

Os dados de precipitação pluviométrica e temperatura do ar foram obtidos junto a Estação Meteorológica do Instituto Agronômico do Paraná (IAPAR), distante aproximadamente quatro quilômetros do local da pesquisa.

Os dados obtidos foram analisados através de médias mensais, análise de variância complementada por Tukey em blocos; Tukey mais fatorial em blocos com comparação dos meses e correlação linear entre as espécies encontradas em relação à temperatura e precipitação durante análise de um ano de coleta.

\section{RESULTADOS E DISCUSSÃO}

Entre larvas e pupas de Culicidae foram coletadas e analisadas 12.656 exemplares, pertencentes a seis gêneros e 11 espécies diferentes. A tabela I lista e quantifica as espécies coletadas nos diferentes tipos de criadouros artificiais.

Nos pneus foram coletados $88,7 \%$ do total de larvas analisadas e das 81 repetições, um único pneu não esteve colonizado por Culicidae durante todo o experimento.

Pneus têm se caracterizado como criadouro preferido por muitas espécies de mosquitos. Segundo BEIER et al. (1983), a preferência por pneus pode ser explicada pela semelhança desses artefatos com ocos de árvore. LOPEs (1997a, b) e Lopes et al. (1993) encontraram este mesmo resultado quando instalaram recipientes em mata ciliar, analisaram outros encontrados no peridomicílio e também na área urbana. Somente Culex bigoti Bellardi, 1862 e Trichoprosopon compressum Lutz, 1905, não colonizaram os pneus. Trichoprosopon Theobald, 1901 caracteriza-se como mosquito silvestre e é coletado com freqüência em internódios de bambu (CERQueira 1961, Lozovei \& Luz 1976), ocos de árvore, cascas de frutas caídas, axilas de folhas e bromélias (Foratrinı \& MARques 2000), o que pode explicar a ausência de suas larvas nos pneus. Todavia larvas de Culex bigoti já foram coletada em pneus por Lopes (1997a, b), na área rural de Londrina e Região.

Pneus podem abrigar grande densidade específica (BeIER et al. 1983). A maior abundância foi observada em um pneu, analisado na segunda metade do mês de novembro de 1995, que continha 911 imaturos de culicídeos. A maior diversidade específica foi constatada em um pneu que continha apenas 54 larvas, analisado na segunda quinzena de janeiro de 1996, onde identificou-se sete espécies coabitando: Limatus durhami Theobald, 1901, Culex eduardoi Casal \& Garcia, 1968, Aedes albopictus (Skuse, 1894), Aedes terrens (Walker, 1856), Aedes

Revista Brasileira de Zoologia 22 (3): 656-661, setembro 2005 
Tabela I. Espécies de Culicidae coletadas em pneus e internódios-armadilhas de bambu instalados no interior de uma mata na área urbana de Londrina, Paranaá, de setembro de 1995 a agosto de 1996.

\begin{tabular}{lcccccccc}
\hline \multicolumn{1}{c}{ Espécies } & Pneu & $\%$ & Bambu solo & $\%$ & Bambu alto & $\%$ & Total bambu & Total geral \\
\hline Aedes albopictus & 561 & 5,00 & 119 & 14,03 & 70 & 11,97 & 189 & 750 \\
Aedes aegypti & 78 & 0,70 & - & - & - & - & - & 78 \\
Aedes terrens & 1151 & 10,26 & 217 & 25,59 & 106 & 18,12 & 323 \\
Culex bigoti & - & - & 5 & 0,59 & - & - & 5 & 1474 \\
Culex eduardoi & 1776 & 15,82 & 123 & 14,50 & - & - & 123 \\
Culex mollis & 164 & 1,46 & - & - & - & - & - & 1899 \\
Culex quinquefasciatus & 780 & 6,95 & 117 & 13,80 & 78 & 13,33 & 195 \\
Culex saltanensis & 2 & 0,02 & - & - & - & - & - & 975 \\
Haemagogus leucocelaenus & 118 & 1,05 & 178 & 20,99 & 154 & 26,32 & 332 \\
Limatus durham & 6593 & 58,75 & 82 & 9,67 & 147 & 25,13 & 229 \\
Trichoprosopon compressum & - & - & 7 & 0,83 & 30 & 5,13 & 37 \\
\hline Total & 11223 & 100 & 848 & 100 & 585 & 100 & 1433 \\
\hline
\end{tabular}

aegypti (Linnaeus, 1762), Culex mollis Dyar \& Knab, 1906 e Culex saltanensis Dyar, 1928. Para o mesmo tipo de criaduro, Lopes (1997b) encontrou a coexistência de seis espécies, e em caixa d'água, oito espécies de culicídeos foram capazes de coexistirem (Lopes 1997a). Neste experimento, a freqüência maior foi a ocorrência de mais que uma espécie colonizando o criadouro. Este resultado está de acordo com BEIER et al. (1983), quando afirmaram que várias espécies podem coabitar mas apenas uma ou duas predominam. Neste trabalho verificou-se a predominância de Limatus durhami; todavia também foram freqüentes e abundantes Culex eduardoi, Culex quinquefasciatus e Aedes terrens. Na área urbana de Macau - Ásia, EASTON (1994) constatou 15 espécies de Culicidae coabitando, observando predominância de três: Culex quinquefasciatus, Culex sitiens Wiedemann, 1828 e Aedes albopictus.

Pelas tabelas I e II observa-se que Limatus durhami foi a espécie mais abundante do pneu (58,75\%), Aedes terrens nos bambus instalados ao nível do solo $(25,59 \%)$ e nos bambus instalados a dois metros de altura Haemagogus leucocelaenus (Dyar \& Shannon, 1924) (26,78\%) e Limatus durhami (25,57\%) foram as mais abundantes.

Culex quinquefasciatus, Aedes terrens, Aedes albopictus, Haemagogus leucocelaenus e Limatus durhami. mostraram possuir valência ecológica que os capacitam colonizar as três condições de criadouros.

Em estudo na área urbana e periurbana na região de Londrina, Lopes et al. (1993) observaram que Limatus durhami foi mais freqüente nos criadouros localizados em áreas com mais arborização, aumentando a sua incidência, conforme se aproximava da área periurbana, principalmente colonizando criadouros sombreados. As condições do pequeno capão de mata, dentro da área urbana, onde foi montado o presente experimento, oferece condições ideais para a procriação desta espécie, que mostra-se adaptada a colonizar recipientes artifi- ciais. Foi ativa nos meses de temperatura mais elevada com densidade populacional máxima em abril e densidade mínima nos meses de julho a setembro (Fig. 1) e CV $=49,62 \%$, o qual mostra a eficácia do teste empregado nessa situação. Um experimento realizado na área rural dos Municípios de Cambé e Londrina, Paraná. Lopes (1997a) mostrou que Limatus durhami também foi a mais abundante no período do verão, juntamente com Culex quinquefasciatus e Culex eduardoi.

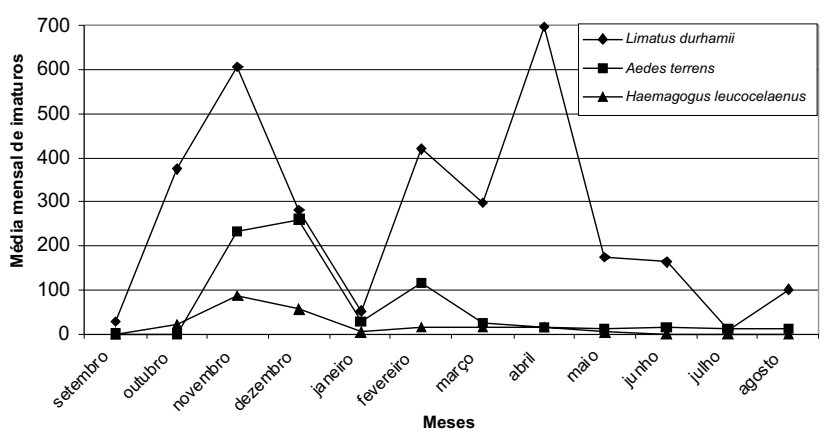

Figura 1. Média mensal de três espécies de Culicidae encontradas em criadouros de pneu e internódio - armadilha de bambu instalados no interior da mata Daher, Londrina, Paraná. Médias originais. Para o teste de Tukey os dados foram transformados em raiz de $x+1$.

Todas as espécies coletadas tiveram diminuição de suas densidades populacionais nos meses mais frios do ano (Fig. 1). Esta influência direta da temperatura sobre a atividade dos Culicidae é amplamente conhecida, assim como o aumento populacional nos períodos mais quentes e chuvosos do ano. Em janeiro houve uma situação atípica quando foi registrada queda 
Tabela II. Total anual para as seis espécies de Culicidae mais freqüentes encontradas durante um ano de coleta em três tipos diferentes de criadouros instalados no interior da mata Daher, Londrina, Paraná e as associações resultantes do teste de Tukey.

\begin{tabular}{lcccccc}
\hline Criadouros & Limatus durham & Culex eduardoi & Culex quinquefasciatus & Aedes albopictus & Aedes terrens & Haemagogus leucocelaenus \\
\hline Pneus & $6593 \mathrm{a}$ & $1776 \mathrm{a}$ & $780 \mathrm{a}$ & $561 \mathrm{a}$ & $1151 \mathrm{a}$ & $118 \mathrm{a}$ \\
Bambus alto & $147 \mathrm{~b}$ & $0 \mathrm{~b}$ & $78 \mathrm{a}$ & $70 \mathrm{a}$ & $106 \mathrm{~b}$ & $154 \mathrm{a}$ \\
Bambus solo & $82 \mathrm{~b}$ & $123 \mathrm{ab}$ & $117 \mathrm{a}$ & $119 \mathrm{a}$ & $217 \mathrm{~b}$ & $178 \mathrm{a}$ \\
CV\% & 28,9 & 50,38 & 38,62 & 20,86 & 12,17 & 4,41 \\
\hline
\end{tabular}

* Dados originais totais seguidos da mesma letra na coluna não diferem entre si pelo teste de Tukey no nível de $5 \%$ de significância. Para o teste, os dados foram transformados em raiz de $x+1$.

drástica nas densidades populacionais das espécies estudadas, sem uma causa aparente ou explicável (Fig. 1) e CV = 58,41\%.

Aedes terrens é um mosquito que procria-se preferencialmente em ocos de árvores (Lozover \& Luz 1976, Neves \& Faria 1997), embora tenha sido coletado com freqüência em criadouros artificiais, no norte do Paraná, por Lopes (1997a). Até 1988, não aparecia em coletas sistemáticas realizadas em criadouros naturais ou artificiais na região norte do Paraná, quando foi registrado por Lopes (1997a). Em observações subsequentes, passou a ser então uma espécie relativamente comum nas coletas de larvas em criadouros artificiais e naturais em áreas degradadas (Lopes 2002). Esta espécie mostrou preferência para ovipor em pneus (Tab. II), e neste tipo de criadouro, sua variação populacional apresentou correlação significativa com a variação da temperatura, comprovada teste de correlação linear, ao nível de 5\% ( $=0,0598)$, mas não foi significativo com as condições de precipitação pluviométrica. As maiores médias populacionais ocorreram em novembro e dezembro (Fig. 1), no final da primavera e início do verão. Este mesmo tipo de atividade foi constatado por Lopes (1997a) em coletas de imaturos em bambu e pneus instalados em mata ciliar em Cambé e Londrina, Paraná.

Das 81 coletas realizadas, Aedes albopicutus foi observada em 44 vezes no pneu, em 12 vezes nos recipientes de bambu instalados ao nível do solo e apenas quatro vezes nos bambus instalados a dois metros de altura. Esta ampla ocupação de criadouros está de acordo com a conhecida valência ecológica, que o capacita colonizar ambientes silvestres e antrópicos e fazer postura tanto em criadouros naturais quanto artificiais. Este comportamento dificulta as ações de controle. Algumas pesquisas têm mostrado a superioridade competitiva desta espécie sobre outras, inclusive sobre as nativas (Juliano 1998).

Aedes aegypi foi coletada em pneus em cinco oportunidades. Em quatro ocasiões coexistia com Aedes albopictus e em duas estava também presente Haemagogus leucocelaenus. A preferência de Aedes aegypti por pneu e a sua baixa freqüência, está de acordo com seu hábito de mosquito urbano. Todavia esta pequena reserva pode servir como refúgio para a espécie e, desta forma, as campanhas de controle do mosquito transmissor do dengue não podem deixar de considerar esse tipo de habitat, o que torna as atividades mais problemáticas.

Haemagogus leucocelaenus, mosquito vetor da febre amarela, foi coletada em nove oportunidades coexistindo com Aedes albopictus em recipiente de pneu. Larvas de Haemagogus leucocelaenus, apareceram 12 vezes nos pneus, 13 vezes nos bambu do solo e 17 vezes nos internódios-armadilhas de bambu instalados a dois metros de altura; não havendo diferença estatística quanto à preferência pelo criadouro (Tab. II). O registro de larvas desta espécie em pneus foi feito por Lopes (1997a). Essa espécie teve picos populacionais em novembro e dezembro (Fig. 1) e CV = $58,69 \%$, semelhante ao encontrado por BARBosa et al. (2003), em coletas com isca humana na cidade de Curitiba, Paraná.

Culex quinquefasciatus é característico de criadouros com muita matéria orgânica, porém, colonizou todos os tipos de criadouros ofertados (Tab. II). Para essa espécie houve uma correlação positiva com a precipitação no criadouro de bambu no solo ( $p=0,0233)$. Esta espécie é amplamente conhecida por ser fortemente antrópica.

Culex mollis e Culex saltanensis foram coletadas somente em pneu. A primeira espécie é mais comum em criadouros que contenham muita matéria orgânica ou poças com vegetação e a última, embora coletada em caixa d'água Lopes (1997b), é comumente encontrada em lagoas altamente poluídas. Culex saltanensis foi encontrada pela primeira vez no Rio de Janeiro em capturas de adulto por LouRENÇO-DE-OLIVEIRA (1984) sendo em seguida, encontrada de forma ininterrupta através de coletas com isca humana em Granjas Calábria, Rio de Janeiro, com abundância no final da primavera e verão (LourEnÇO-DE-OliveIRA et al. 1985). Esta espécie poder ser um potencial vetor de agentes etiológicos, já que se infecta com Plasmodium catherimerium hemosporídeo de pardais (GABALDON et al. 1988) e foi considerado como vetor primário de Plasmodium juxtanucleare no Rio de Janeiro (LourenÇO-DE-Oliveira \& CASTro 1991). Sibajev et al. (1993) descreveram uma nova espécie de tripanossomatídeo - Critidia ricardoi considerado hospederio original de Culex saltanensis.

Das 11 espécies coletadas, Culex quinquefasciatus, Aedes aegypti e Aedes albopictus são características de áreas antrópicas, fortemente antropofílicas e procriam-se preferencialmente em recipientes artificiais (LOPES 2002). Aedes terrens e Haemagogus leucocelaenus são culicideos considerados de hábitos silvestres, mas que atualmente estão sendo coletados em criadouros naturais e artificiais em área antrópica, todavia guardando associação com a presença de vegetação e condições de sombreamento (Lourenço-De-Oliveira et al. 1986, Lopes 1997a, b). Esta mesma associação é constatada para Limatus durhami, porém

Revista Brasileira de Zoologia 22 (3): 656-661, setembro 2005 
esta se faz mais freqüente e abundante, mostrando estágio mais avançado na questão evolutiva adaptativa às condições antrópicas (LOPEs 2002). Culex bigoti espécie considerada silvestre tem sido freqüente, mas pouco abundantes em coletas realizadas em recipientes e poças d'água nas regiões urbana e rural (Lopes 1997a, b). Culex eduardoi e Culex mollis são espécies encontradas em área silvestre, mas também, e com maior freqüência, em ambientes alterados, colonizando criadouros preferencialmente com vegetação e muita matéria orgânica, mas com valência ecológica para colonizar recipientes artificiais comuns em área urbana e rural (LOPEs 1997a, b). Culex saltanensis, mosquito comum em área alterada pelo homem, sendo observada competindo com Culex quinquefasciatus em lagoas de tratamento de efluentes (Lopes 2002). Trichoprosopon compressum foi a espécie que apresentou maiores características silvestres, principalmente relacionadas às exigências reprodutivas e com potencial para colonizar fragmento florestal na área urbana.

Mata ciliar localizada na área urbana pode servir como local de repouso e ou criação de várias espécies de Culicidae. O seu potencial como local de criação aumenta pela ação do homem, quando este usa indevidamente estas áreas para descarte de objetos que possam reter água. Desta forma, medidas de vigilância, prevenção e controle não podem ignorar esses habitats, principalmente por ter sido registrado a presença de Aedes aegypti, Aedes albopictus e Haemagogus leucocelaenus, três espécies consideradas como vetoras do dengue e febre amarela na área urbana.

\section{AGRADECIMENTOS}

Aos docentes Dra Dileimar Machado Nalim Gallegos do Departamento de Biologia Animal e Vegetal e ao Dr. Maurício Ursi Ventura do Departamento de Agronomia, Universidade Estadual de Londrina pelas revisões no artigo. Ao Programa PIBIC/CNPq pela bolsa de Iniciação Científica.

\section{REFERÊNCIAS BIBLIOGRÁFICAS}

Barbosa, A.A.; M.A.N. Silva; D. Calado. 2003. Atividade de Culicidae em remanescente florestal na região urbana de Curitiba (Paraná - Brasil). Revista Brasileira de Zoologia, Curitiba, 20 (1): 59-63.

Barbosa, O.C.; T. Ueslei; A.L. Lozovei. V.S. Filho; R.P. Spinosa; E.M. Lima \& M.E.M.C. Ferreira. 1993. Nota sobre culicídeos adultos coletados na região sul do Brasil. Revista de Saúde Pública, São Paulo, 27 (3): 214-216.

Beier, J.C.; C. Patricoski; M. Travis. 1983. Influence of water chemical and environment parameters on larval mosquito dynamics in tires. Environonmental Entomology, College Park, 12 (2): 435-438.

Calado, D.C. \& M.A.N. Silva. 2001. Comunidade de mosquitos (Diptera, Culicidae) em recipientes introduzidos em área rural e urbana da região metropolitana de Curitiba, Paraná, Brasil. Revista Brasileira de Zoologia, Curitiba, 18 (Supl.1): 51-60.
Cerqueira, N. L. 1961. Distribuição geográfica dos mosquitos da amazônia. Revista Brasileira de Entomologia, São Paulo, 10: 111-168.

Consolim, J.; N.J.M. Pellegrini; E. Luz. 1993. Culicídeos (Diptera: Culicidae) do Lago Itaipú, Paraná, Brasil. I. Município de Foz do Iguaçu. Acta Biológica Paranaense, Curitiba, 22 (14): 83-90.

EAston, E.R. 1994. Urbanization and its effects on the ecology of mosquitoes in Macau, Southeast - Asia. Journal American Mosquito Control Association, Fresno, 10 (4): 540-544.

Forattinı, O.P. 2002. Culicidologia Médica. São Paulo, Edusp, $864 p$.

Forattini, O.P.; I. Kakitani; E. Massad \& D. ManucCi. 1993. Studies on mosquitoes (Diptera: Culicidae) and antropic environment. 4 - Survey of resting adults and synantropic behaviour in South - Eastern, Brazil. Revista de Saúde Pública, São Paulo, 27 (6): 398-411.

Forattini, O.P.; G.R.A.M. MARQues. 2000. Nota sobre encontro de Aedes aegypti em bromélias. Revista de Saúde Pública, São Paulo, 34 (5): 543-544.

Gabaldon, A.; G. Ulloa \& N. Zerpa. 1988. Plasmodium cathermerium, cepa de Icteridae inoculable a palomas, patos y pavos; sus vectores y utilidad en enseñanza e investigación. Boletín de la Direccion de Malariologia y Saneamiento Ambiental, Macaray, 28: 53-68.

Guimarães, A.E.; G. Carla; C.M. Lopes; A. Santana \& A.M. Jovita. 2000. Ecologia de mosquitos (Diptera: Culicidae) em área do Parque Nacional da Serra da Bocaina, Brasil. I. Distribuição por habitat. Revista de Saúde Pública, São Paulo, 34 (3): 243-250.

Jenkins, D.W. \& S.J. Carpenter. 1946. Ecology of the tree hole breeding mosquitoes of Neartic North America. Ecological Monographs, Lawrence, 16: 33-47.

JuLiano, S.A. 1998. Species introduction and replacement among mosquitoes: interespecific resource competition or apparent competition? Ecology, Tempe, 1: 255-268.

LANE, J. 1953. Neotropical Culicidae. São Paulo, Ed. Brasil., vols1 e 2, 1112p.

LOPES, J. 2002. Mosquitos (Diptera: Culicidae) da Região do Baixo Tibagi e suas adaptações a ambientes antropogênicos: causas e consequências, 327-351. In: M.E. MEdRI; E. BiAnCHINI; O.A. Shibatta \& J.A. Pimenta (Eds). A Bacia do Rio Tibagi. Londrina, M.E. Medri, 595p.

Lopes, J. 1997a. Ecologia de Mosquitos (Diptera, Culicidae) em criadouros naturais e artificiais de área rural do norte do estado do Paraná, Brasil. VI. Coletas de larvas no peridomicílio. Revista Brasileira de Zoologia, Curitiba, 14 (3): 571-578.

Lopes, J. 1997b. Ecologia de mosquitos (Diptera: Culicidae) em criadouros naturais e artificiais de área rural do norte do estado do Paraná, Brasil. V. Coletas de larvas em recipientes artificiais instalados em mata ciliar. Revista de Saúde Pública, São Paulo, 31 (4): 370-377. 
Lopes, J. \& A.L. Lozovei. 1995. Ecologia de mosquitos (Diptera: Culicidae) em criadouros naturais e artificiais de área rural do Norte do Estado do Paraná, Brasil. I - Coletas ao longo do leito do ribeirão. Revista de Saúde Pública, São Paulo, 29 (3): 183-191.

Lopes, J.; M.A.N. Da Silva; A.M. Borsato; V.D.R.B. Oliveira \& F.J. DE A.A. Oliveira. 1993. Aedes (Stegomyia) aegypti L. e a culicideofauna associada em área urbana da região sul, Brasil. Revista de Saúde Pública, São Paulo, 27 (5): 326-333.

Lopes, J.; J.A.C. Zequi; V. Nunes; O. Oliveira; B.P.O. Neto \& W. RoDRIGUES. 2002. Immature Culicidae (Diptera) collected from the Igapó lake located in the urban area of Londrina, Paraná, Brazil. Brazilian Archives of Biology and Technology, Curitiba, 45 (4): 465-471.

Lourenço-De-Oliveira, R. 1984. Alguns aspectos da Ecologia dos mosquitos (Diptera: Culicidae de uma área de planície (Granjas Calábria), em Jacarepaguá, Rio de Janeiro. I. Freqüência comparativa das espécies em diferentes ambientes e métodos de coleta. Memórias do Instituto Oswaldo Cruz, Rio de Janeiro, 79 (4): 479-490.

Lourenço-De-Oliveira, R. \& F.A. Castro. 1991. Culex saltanensis Dyar, 1928. Natural vector of Plasmodium juxtanucleare in Rio de Janeiro, Brasil. Memórias do Instituto Oswaldo Cruz, Rio de Janeiro, 86: 87-94.

Lourenço-De-Oliveira, R.; T.F. da Silva \& R. Heyden. 1985. Alguns aspectos da ecologia dos mosquitos (Diptera: Culicidae), de uma área de planície (Granjas Calábria), em Jacarepaguá, Rio de Janeiro. II. Freqüência mensal e no ciclo lunar. Memórias do Instituto Oswaldo Cruz, Rio de Janeiro, 80 (2): 123-134.

Lourenço-De-Oliveira, R.; R. Heyden \& T.F. DA Silva. 1986. Alguns aspectos da ecologia dos mosquitos (Diptera: Culicidae), de uma área de planície (Granjas Calábria), em Jacarepaguá, Rio de Janeiro. V. Criadouros. Memórias do Instituto Oswaldo Cruz, Rio de Janeiro, 81(3): 265-271.

Lozover, A.L. 1998. Mosquitos dendrícolas (Diptera, Culicidae) em internódios de taquara da floresta atlântica, serra do mar e do primeiro planalto, Paraná, Brasil. Brazilian Archives of Biology and Technology, Curitiba, 41: 501-508.

Lozover, A.L. \& E. Luz 1976. Diptera Culicidae em Curitiba e arredores. I. Ocorrência. Archives of Biology and Technology, Curitiba, 19: 25-42.

Neves, D.P. \& A.C. FARIA. 1997. Biologia do Aedes (Finlaya) terrens (Walker) em condições de campo e laboratório (Diptera, Culicidae). Revista Brasileira de Biologia, Rio de Janeiro, 37 (4): 803-806.

Paterno, U. \& C.B. Marcondes. 2004. Mosquitos antropofílicos de atividade matutina em Mata Atlântica, Florianópolis -
S.C. Revista de Saúde Pública, São Paulo, 38 (1): 133-135. Quintero, L.O.; D.T. Bedsy \& P.T. Wanderli. 1996. Biologia de anofelinos amazônicos. XXI. Ocorrência de espécies de Anopheles e outros culicídeos na área de influência da hidrelétrica de Balbina - cinco anos após o enchimento do reservatório. Acta Amazonica, Manaus, 26 (4): 281-296.

Sibajev, A.; R.S. Pacheco; M.J. Soares; E. Cupolillo; A.B. Santos \& H. Momen. 1993. Crithidia ricardoi sp. n. a new species of trypanossomatidae isolated from Culex saltanensis Dyar, 1928 (Diptera, Culicidae). Memórias do Instituto Oswaldo Cruz, Rio de Janeiro, 88: (4): 541-545.

Silva, M.A.N. \& A.L. Lozover. 1996. Criadouros de mosquitos (Diptera, Culicidae) introduzidos em mata preservada na área urbana de Curitiba, Paraná, Brasil. Revista Brasileira de Zoologia, Curitiba, 13 (4): 1023-1042.

Silva, M.A.N. \& A.L. Lozover. 1998. Mosquitos (Diptera, Culicidae) capturados com isca humana em área preservada de Curitiba, Paraná. Revista Brasileira de Zoologia, Curitiba, 15 (4): 965-976.

Silva, M.A.N.\& A.L. Lozover. 1999. Ocorrência de Haemagogus (Conopostegus) leucocelaenus (Dyar \& Shannon) e Toxorhynchites (Lynchiella) theobaldi (Dyar\& Knab) em ocos de árvores em capão de mata, Curitiba, Paraná, Brasil. Revista Brasileira de Zoologia, Curitiba, 16 (Supl. 1): 257-267.

Silva, M.A.N.; A.A.Barbosa; D. Calado. 2004. Atividade de Mansonia spp. (Mansoniini, Culicidae) em fragmento florestal na área urbana de Curitiba, Paraná, Brasil. Revista Brasileira de Zoologia, Curitiba, 21 (2): 243-247.

Sota, T. \& M. Mogr. 1996. Species Richness and altitudinal variation in the aquatic metazoan community in bamboo phytotelmata from north Sulawesi. Researches on Population. Ecology, Tokyo, 38 (2): 275-281.

Sota, T.; M. Mogi; E. Hayamizu. 1994. Habitat stability and the larval mosquito, community in treeholes and other containers on a temperature island. Researches on Population Ecology, Tokyo, 36 (1): 93-104.

TAIPE-Lagos, C.B. \& D. NATAL. 2003. Culicidae mosquito abundance in a preserved metropolitan area and its epidemiological implications. Revista de Saúde Pública, São Paulo, 37 (3): 275-279.

Urbinatti, P.R.; S. Sendacz \& D. Natal. 2001. Imaturos de mosquitos (Diptera: Culicidae) em parque de área metropolitana aberta a visitação. Revista de Saúde Pública, São Paulo, 35 (5): 461-466.

Zequi, J.A.C. \& J. Lopes. 2001. Culicideofauna (Diptera) encontrada em entrenós de taquara de uma mata residual na área urbana de Londrina, Paraná, Brasil. Revista Brasileira de Zoologia, Curitiba, 18 (2): 429-438.

Recebido em 17.XI.2004; aceito em 10.VIII.2005. 